\title{
2016 CSHP National Awards Program Winners Programme national des prix 2016 de la SCPH : lauréats et lauréates
}

The winner of the Distinguished Service Award (sponsored by Johnson \& Johnson, Family of Companies) is Donald Kuntz (Regina, SK).

The winner of the Isabel E. Stauffer Meritorious Service Award (sponsored by Fresenius Kabi Canada Ltd.) is Carolee Awde (Peterborough, ON).
The winners of the New Hospital Pharmacy

Practitioner Award (sponsored by Sandoz Canada Inc.) are Olivia Gayan Ng (Toronto, ON) and Ashley N Walus (Winnipeg, MB).

The winner of the Hospital Pharmacy Student Award (co-sponsored by the Canadian Society of Hospital Pharmacists [CSHP] and the Canadian Association of Pharmacy Students and Interns [CAPSI]) is Lydia Cheung (Edmonton, $A B$ ).
Management and Leadership Best Practice Award Sponsored by Apotex Inc.

Development of a Pharmacist Performance Tracking Tool (completed at Royal University Hospital)

Alyssa MacDougall, Barb Evans

Sponsored by Medbuy Corporation

Is Your Turnaround Time Fast Enough? (completed at Montfort Hospital)

Lise Vaillancourt

Patient Care Enhancement Award Sponsored by Pfizer Canada Inc.

The Medication Assessment Centre: A Novel Student Training and Patient Care Program (completed at College of Pharmacy and Nutrition, University of Saskatchewan) Derek Jorgenson, Eric Landry, Jaris Swidrovich, Katherine Lysak Sponsored by Teva Canada Limited

Prescribing Patterns of Hospital Pharmacists with Additional Prescribing Authorization in Alberta (oral presentation) (completed at University of Alberta Hospital)

Taryn Heck, Lauren Bresee, Sheri Koshman, Tania Mysak, Mary Gunther

Pharmacotherapy Best Practices Award Sponsored by Pfizer Canada Inc.

Development of Clinical Pharmacy Key Performance Indicators for Hospital Pharmacists Using a Modified Delphi Approach (completed at University Health Network) Olavo Fernandes, Sean K Gorman, Richard S Slavik, William M Semchuk, Stephen Shalansky, Jean-François Bussières, Douglas Doucette, Jennifer Lo, Winnie W Y Chan, Natalie Benninger, Neil J MacKinnon, Jeremy Slobodan, Catherine Lyder, Peter I Zed, Kent Toombs

Sponsored by Sandoz Canada Inc.

Pediatric Assessment of Vancomycin Empiric Dosing 2 (PAVED2) (completed at British Columbia Children's Hospital)

Kyle Collins, Mary H H Ensom, Daniel Rainkie, Roxane Carr
Safe Medication Practices Award

Sponsored by HealthPRO Procurement Services Inc. Quality Assurance of Aseptic Compounding in Winnipeg Regional Health Authority Pharmacies (oral presentation) (completed at Winnipeg Regional Health Authority)

Donna M M Woloschuk, Cenzina Caligiuri

Sponsored by Medbuy Corporation

Pilot Study of Biological Monitoring of Four Antineoplastic Drugs among Canadian Healthcare Workers (completed at CHU Sainte-Justine)

Jean-François Bussières

Specialties in Pharmacy Practice Award Sponsored by Pharmascience Inc.

Extended-Interval Dosing Recommendations for Gentamicin in Neonates (completed at Sunnybrook Health Sciences Centre)

Monique Potvin, Sandra A N Walker, Marion Elligsen,

Dolores Iaboni, Winnie Seto

Sponsored by Sandoz Canada Inc.

Acute Kidney Injury with Tobramycin-Impregnated

Bone Cement Spacers in Prosthetic Joint Infections

(completed at Vancouver General Hospital)

Elissa S Y Aeng, Karen F Shalansky, Tim T Y Lau

Teaching, Learning and Education Award

Sponsored by Eli Lilly Canada Inc.

Learning from Medication Incidents One Group at a Time: The Development and Evaluation of Multi-Incident Analysis Workshops (oral presentation) (completed at Institute for Safe Medication Practices Canada)

Roger Cheng, Sylvia Hyland

The award-winning abstracts are published exactly as submitted by the author and have not undergone any copyediting by the Canadian Journal of Hospital Pharmacy.

Le Journal canadien de la pharmacie hospitalière n'a pas soumis les résumés primés à une révision linguistique et les publie ici tels que remis par les auteurs. 


\section{Development of a Pharmacist Performance Tracking Tool}

\section{Management and Leadership Best Practice Award, sponsored by Apotex Inc.}

MacDougall A, Evans B

Saskatoon Health Region, Saskatoon, SK

Introduction: Many pharmacist activities have been demonstrated to positively impact patient flow, reduce morbidity and mortality, and decrease medication errors and adverse drug reactions. This evidence, and organizations' goals, should be considered when defining a pharmacists' best-practice model. Development of a pharmacists' performance tracking tool will allow for measurement of these key performance indicators and assist in advancing pharmacy practice. The purpose of this study was to develop an evidence-based, pharmacist performance tracking tool to capture key performance indicators that positively impact patient outcomes.

Methods: The study was conducted on Pediatric and Medicine units at Royal University Hospital in the Saskatoon Health Region. It consisted of four stages: literature review, focus groups with staff pharmacists, consultations with experts, and three Plan-Do-Check-Act cycles. Completed key performance indicators were tallied, and those completed during regular care were compared to the new Model of Care practice.

Results: An evidence-based Pharmacist Daily Performance Tracking Tool was developed and included eleven key performance indicators. The most frequently completed key performance indicator was identification and resolution of drug therapy problems. Significantly more drug therapy problems were addressed on the Model of Care practice where a smaller patient to pharmacist ratio existed.

Discussion: Measurement of key performance indicators demonstrated pharmacist commitment to the Health Regions' operational goals. Obtaining drug coverage for patients facilitated timely discharge and contributed to the organizational goal of improved patient flow. With a smaller patient to pharmacist ratio, there was more time for direct patient care and drug therapy review which resulted in the identification and resolution of significantly more drug therapy problems.

Conclusions: A Pharmacist Daily Performance Tracking Tool facilitated measurement of pharmacist activities known to improve patient outcomes. Focusing on key performance indicators can push hospital pharmacists towards an evidence-based practice model.

\section{Is Your Turnaround Time Fast Enough? \\ Management and Leadership Best Practice Award, sponsored by Medbuy Corporation \\ Vaillancourt L, Truong $M$ \\ Montfort Hospital, Ottawa, ON}

Introduction: Montfort Hospital has doubled its size in the past years and has worked to decrease its length of stay in acute beds and the number of alternate level of care patients. This has had an enormous impact on the number of new orders received in pharmacy. Our department was having difficulty to cope with this increased workload and sending new medication to the nursing units in a timely fashion.

Description: The pharmacy team used some LEAN techniques (work cell production system, visual management, capacity and demand and Little's Law) to improve its turnaround time for a new order.
Outcomes: Using LEAN Principles, the pharmacy staff at Montfort Hospital was able to rapidly decrease its turnaround time for a new prescription by approx. $40 \%$ from an average of 2:34 hours to 1:30 hour. The implication of staff in this project also helped decrease the overtime to an average of less than 25 hours per month.

Discussion: The team had been trying for a few years to improve its client satisfaction, turnaround time and overtime. It seems like this innovative approach provides results and was also useful in building a stronger team.

Conclusion: The use of LEAN methodology is innovative in pharmacy practice and can help teams to become more efficient.

\section{The Medication Assessment Centre: A Novel Student Training and Patient Care Program \\ Patient Care Enhancement Award, sponsored by Pfizer Canada Inc.}

\section{Jorgenson D, Landry E, Swidrovich J, Lysak K}

College of Pharmacy and Nutrition, University of Saskatchewan, Saskatoon, $S K$

Introduction: The Medication Assessment Centre was launched in 2014 by the College of Pharmacy and Nutrition at the University of Saskatchewan, with the dual purposes of: (1) providing access to pharmacist-led comprehensive medication management for complex patients who cannot access the service elsewhere; and, (2) creating an early experiential training program for pharmacy students. The purpose of this project is to describe the Medication Assessment Centre and to report on results from its program evaluation.

Description of the Project: The Medication Assessment Centre is a pharmacist-run clinic located in Saskatoon, Saskatchewan that provides comprehensive medication management services for complex patients. Pharmacy students from all four years of the University of Saskatchewan pharmacy program volunteer at the Medication Assessment Centre using a 4:1 student - pharmacist ratio, a peer training model, and reflective learning strategies.

Project Outcomes: Student volunteer opportunities have been popular and a total of 68 students volunteered regularly during the first year. The Medication Assessment Centre currently follows 312 highly complex patients on a regular basis, referred from both health professionals $(62.5 \%)$ and patients themselves (37.5\%). Patient mean age is 71 and they have, on average, 9.2 diagnoses and take 13.3 medications each. The Medication Assessment Centre's pharmacists make, on average, 6.0 recommendations per patient ( $23.0 \%$ for problems of moderate severity). Ninety experience questionnaires were mailed to patients, and 65 to physicians, between April and July 2015 and responses have been very supportive and positive.

Discussion: Program evaluation is limited by the fact that the Medication Assessment Centre has only been open for 1.5 years. Nevertheless, existing data are sufficient to justify continuing the program.

Conclusion: The Medication Assessment Centre has achieved its dual purposes and it will consequently be integrated into the new PharmD Curriculum at the College of Pharmacy and Nutrition, University of Saskatchewan, in 2017. 


\section{Prescribing Patterns of Hospital Pharmacists with Additional Prescribing Authorization in Alberta \\ Patient Care Enhancement Award, sponsored by Teva Canada Limited \\ Heck $T^{1}$, Bresee L L,3, Koshman S4, Mysak T', Gunther $M^{1}$ \\ ${ }^{1}$ Pharmacy Services, Alberta Health Services, Edmonton, $A B$ \\ ${ }^{2}$ Pharmacy Services, Alberta Health Services, Calgary, $A B$ \\ ${ }^{3}$ Faculty of Medicine, University of Calgary, Calgary, $A B$ \\ ${ }^{4}$ Division of Cardiology, University of Alberta, Edmonton, $A B$}

Introduction: Alberta Health Services has established a vision for clinical pharmacy practice that supports pharmacists working to their full scope of practice, which includes independent prescribing. However, information regarding prescribing patterns of hospital pharmacists with additional prescribing authorization (APA) is lacking. The objective of the study was to determine the self-reported frequency and characteristics of prescribing by hospital pharmacists with APA who provide clinical pharmacy services within inpatient and/or outpatient settings.

Methods: Between January and March 2014, we conducted a descriptive, cross-sectional study utilizing web-based, self-enumerated survey data. Pharmacists employed within inpatient and/or outpatient settings, and granted APA as of December 31, 2013, were eligible. Descriptive statistics and logistic regression analysis were used to report outcomes.

Results: The median frequency of prescriptions written per 10 patients during an average week was 4.2 (IQR 8.0). The most frequently completed prescribing activities were following team discussion (1.8 [IQR 2.5]) and adjusting dose based on organ function and clinical assessment (1.0 [IQR 2.3]). The leading barrier and enabler for prescribing overall was interdisciplinary care team dynamics.

Discussion: This study represents the first attempt to quantify the frequency of prescribing among hospital pharmacists with APA. Hospital pharmacists with APA prescribe for almost half of their patients, which suggests that pharmacists are utilizing their prescribing rights, in addition to other skills and knowledge, on a regular basis to provide patient care. Collaboration was a key consideration of pharmacist prescribers, supporting that pharmacists can still influence prescribing to improve patient care in jurisdictions where independent prescribing is unattainable.

Conclusion: The results of the study reflect the positive uptake of practicing to full scope in hospital pharmacy and the ongoing importance of collaboration in the hospital setting.

\section{Development of Clinical Pharmacy Key Performance Indicators for Hospital Pharmacists Using a Modified Delphi Approach}

\section{Pharmacotherapy Best Practices Award, sponsored} by Pfizer Canada Inc.

Fernandes $O^{1,2}$, Gorman $S K^{3,4}$, Slavik $R S^{3,4}$, Semchuk WM, Shalansky $S^{4,7}$, Bussières J-F $F^{8,9}$, Doucette $D^{10,11}$, Lo $J^{12}$, Chan WWYY13, Benninger $N^{14}$, MacKinnon $N J^{15}$, Bell $C M^{16,17,18}$, Slobodan $J^{19}$, Lyder $C^{20}$, Zed PI', Toombs $K^{21}$

${ }^{1}$ Pharmacy Department, University Health Network, Toronto, ON ${ }^{2}$ Leslie Dan Faculty of Pharmacy, University of Toronto, Toronto, ON ${ }^{3}$ Interior Health Pharmacy Services, British Columbia

${ }^{4}$ Faculty of Pharmaceutical Sciences, The University of British Columbia, Vancouver, $B C$
${ }^{5}$ Clinical Pharmacy Services, Regina Qu'Appelle Health Region, Regina, SK ${ }^{6}$ College of Pharmacy and Nutrition, University of Saskatchewan, Saskatoon, SK

${ }^{7}$ Pharmacy, Providence Health Care, Vancouver, BC

${ }^{8}$ Département de pharmacie et Unité de recherche en pratique pharmaceutique, CHU Sainte-Justine, Montréal, QC ${ }^{9}$ Faculté de pharmacie, Université de Montréal, Montréal, QC ${ }^{10}$ Pharmacy Services, Horizon Health Network, New Brunswick ${ }^{11}$ College of Pharmacy, Dalhousie University, Halifax, NS

${ }^{12}$ Pharmacy Department, Sunnybrook Health Sciences Centre, Toronto, ON

${ }^{13}$ Pharmacy Department, St Michael's Hospital, Toronto, ON

${ }^{14}$ Pharmacy Department, University Health Network - Toronto Rehabilitation Institute, Toronto, ON

${ }^{15}$ James L. Winkle College of Pharmacy, University of Cincinnati, Cincinnati, $\mathrm{OH}$

${ }^{16}$ Division of General Internal Medicine, Mount Sinai Hospital, Toronto, ON

${ }^{17}$ Institute of Health Policy, Management and Evaluation, University

of Toronto, Toronto, ON

${ }^{18}$ Institute for Clinical Evaluative Sciences, Toronto, ON

${ }^{19}$ Pharmacy Services, Alberta Health Services, Alberta

${ }^{20}$ Canadian Society of Hospital Pharmacists, Ottawa, ON

${ }^{21}$ Pharmacy Department, Capital District Health Authority, Halifax, NS

Introduction: There are no published, systematically-derived clinical pharmacy key performance indicators (cpKPIs). The aim was to develop national cpKPIs to advance clinical pharmacy practice and improve patient care.

Methods: A working group established a cpKPI definition, eight cpKPI critical activity areas, 26 candidate cpKPIs, $11 \mathrm{cpKPI}$ ideal attributes, and one overall consensus criterion. Over a 3-month period, 26 clinical pharmacists and hospital pharmacy leaders participated in a three-round modified Delphi survey. Using an Internet-based, pre-tested survey instrument, panelists independently rated the 26 candidate cpKPIs using the $11 \mathrm{cpKPI}$ ideal attributes and one overall consensus criterion on a nine-point Likert scale. A meeting was facilitated between rounds two and three to debate the merits of each candidate cpKPI. Consensus was reached if $75 \%$ or more of the panelists assigned a score of seven to nine on the consensus criterion during the final round.

Results: All panelists completed the three Delphi rounds and 25/26 (96\%) attended the meeting. Eight candidate cpKPIs reached consensus: 1) performing admission medication reconciliation; 2) participating in inter-professional patient care rounds; 3) completing pharmaceutical care plans; 4) resolving drug therapy problems; 5) providing in-person disease and medication education to patients 6) providing discharge patient medication education; 7) performing discharge medication reconciliation; and 8) providing bundled, proactive direct patient care activities.

Discussion: Strengths of this study were the consensus-making methodology and inclusion of panelists from across Canada with experiences representative of hospital pharmacy practice. A limitation was the inability to reach consensus on drug or disease-specific cpKPIs. Future research should focus on developing drug or disease-specific cpKPIs and on evaluating the real world impact of the cpKPIs on patient outcomes.

Conclusions: A Delphi panel of hospital pharmacists was successful in determining eight consensus cpKPIs. Measurement and assessment of these cpKPIs should advance clinical pharmacy practice and improve patient care. 


\section{Pediatric Assessment of Vancomycin Empiric Dosing 2 (PAVED2) \\ Pharmacotherapy Best Practices Award, sponsored by Sandoz Canada Inc. \\ Collins $K^{1}$, Ensom $M H H^{1,2}$, Rainkie $D^{3}$, Carr $R^{1,2}$ \\ ${ }^{1}$ Children's \& Women's Health Centre of BC, Vancouver, $B C$ \\ ${ }^{2}$ Faculty of Pharmaceutical Sciences, The University of British Columbia, \\ Vancouver, $B C$ \\ ${ }^{3}$ University of British Columbia, Vancouver, $B C$}

Introduction: Our previous study (PAVED) demonstrated therapeutic vancomycin serum concentrations were not achieved using a common empiric dosing regimen. The purpose of this study was to validate the PAVED dosing regimen by retrospectively applying it to a group of patients who received vancomycin, and evaluating whether this regimen more reliably achieved target serum concentrations than the current regimen. Our objectives were to: $i$ ) describe the proportion of patients who would have reached initial target trough concentrations using the PAVED regimen, ii) to describe the characteristics pertaining to dosing and pharmacokinetic parameters, iii) to evaluate the concordance between trough concentrations and area under the curve (AUC).

Description of the Methods: Following ethics approval, 200 patients aged 1 month-18 years who received vancomycin and had at least two pharmacokinetically interpretable serum concentrations were included.

Results: Using British Columbia Children Hospital's (BCCH) current empiric regimen, 110 (55\%) individuals obtained initial vancomycin serum trough concentrations $<10 \mathrm{mg} / \mathrm{L}, 58(29 \%)$ achieved between $10-15 \mathrm{mg} / \mathrm{L}, 27$ (14\%) between 15-20 mg/L, and 5 (2\%) >20 mg/L. Using the regimen recommended by PAVED, a greater proportion of individuals were predicted to achieve target trough concentrations: 65 individuals $(32 \%)<10 \mathrm{mg} / \mathrm{L}, 71(36 \%)$ between $10-15 \mathrm{mg} / \mathrm{L}, 35(18 \%)$ between $15-20 \mathrm{mg} / \mathrm{L}$, and $29(14 \%)>20 \mathrm{mg} / \mathrm{L} .99 \%$ of individuals who achieved a trough concentration $>10 \mathrm{mg} / \mathrm{L}$ also achieved a therapeutic AUC $>400 \mathrm{mg}^{*}$ day/L. Of the individuals who achieved trough concentrations $<10 \mathrm{mg} / \mathrm{L}, 33(27 \%)$ achieved an AUC $>400 \mathrm{mg}^{*}$ day/L and 89 (73\%) achieved an AUC < $400 \mathrm{mg}^{*}$ day/L.

Discussion: BCCH's current empiric regimen achieves subtherapeutic trough concentrations in the majority of patients.

Conclusions: Although a greater proportion of patients are predicted to achieve therapeutic concentrations with the PAVED regimen, it is also predicted to result in a significant number of patients who will achieve supratherapeutic concentrations $>20 \mathrm{mg} / \mathrm{L}$.

\section{Quality Assurance of Aseptic Compounding in Winnipeg Regional Health Authority Pharmacies Safe Medication Practices Award, sponsored by HealthPRO Procurement Services Inc.}

Woloschuk DMM, Caligiuri C Winnipeg Regional Health Authority, Winnipeg, MB

Objectives: We created an effective, sustainable, multi-faceted quality assurance (QA) program that is compatible with contemporary aseptic compounding standards and usable by others.

Description of the Project: QA assessments of aseptic operator training and QA audits of aseptic compounding facilities (ACF) and aseptic operators were developed and implemented. A pharmacy assistant, who was independent of teams being audited, gathered all data and reported it to managers and staff. Education about results promoted adherence to required practices.
Project Experience: Initial training and revalidation at prescribed intervals affected 306 staff and trainers at 16 ACF since 2009. Most trainers $(21 ; 84 \%)$ exhibited no evidence of practice drift during QA assessments. ACF inspections (63) conducted September 2011 to June 2015 resulted in 170 citations of which $44.7 \%$ were mitigated, $8.8 \%$ could only be mitigated by replacing the ACF, and $35.9 \%$ require Maintenance/Housekeeping support plus ACF shutdown for at least 24 hours to remedy. Practice drift among aseptic operators was easily detected by serial QA audits. Audit results informed changes to policy and training materials. QA program operation required 4 paid hours per ACF annually for data collection, analysis, and reporting. The QA program was sustainable at reasonable cost.

Discussion: Complex equipment and stringent operational requirements plus a large workforce that performed aseptic compounding part-time made it challenging to maintain a high level of compliance to standards. Continuous QA monitoring provided a reliable, non-threatening way to identify practice drift and assured prompt attention to equipment and operational deviations.

Conclusion: Sustaining high quality ACF and aseptic operations benefitted from intermittent, unannounced audits conducted by a third party. Other pharmacies can easily and with minimal investment use the audit tools in their aseptic compounding settings.

\section{Pilot Study of Biological Monitoring of Four Antineoplastic Drugs among Canadian Healthcare Workers}

\section{Safe Medication Practices Award, sponsored by Medbuy Corporation}

Poupeau $C^{1}$, Tanguay $C^{1}$, Plante $C^{2}$, Caron N3, Bussières J-F, $F^{1,4,5}$ ${ }^{1}$ Unité de recherche en pratique pharmaceutique, CHU Sainte-Justine, Montréal, QC

${ }^{2}$ Soins infirmiers, CHU Sainte-Justine, Montréal, QC

${ }^{3}$ Centre de toxicologie du Québec, Institut national de santé publique du Québec, Québec, QC

${ }^{4}$ Département de pharmacie, CHU Sainte-Justine, Montréal, QC ${ }^{5}$ Faculté de pharmacie, Université de Montréal, Montréal, QC

Introduction: There are health risks to workers occupationally exposed to antineoplastic drugs.

Purpose/Objectives: We hypothesized that implementing a biological monitoring program would be feasible. The goal is to present the results of our pilot cross-sectional study of biological monitoring of four antineoplastic drugs.

Description of the Projet/Methods: We recruited workers from the hematology-oncology department and control workers from other departments in a mother-child university health center. This study was preceded by an information period during which we aimed at enhancing the workers' awareness and knowledge of the risks of occupational exposure. Participants filled out a journal containing activities performed and personal protective equipment worn. One urine sample was collected at the end of their shift. Samples were analysed by HPLC/MS-MS for the presence of cyclophosphamide, ifosfamide, methotrexate and alpha-fluoro-beta-alanine (5-fluorouracile's main urinary metabolite).

Projet Outcomes/Results: The participation rate was $85.7 \%$ (102/119). No urine sample had detectable concentrations of any of the four drugs evaluated $(0 / 101 ; 0 / 74$ nurses, $0 / 11$ pharmacists, $0 / 9$ pharmacy technicians and $0 / 7$ doctors). In the five days before sampling, 67/89 (75.3\%) hematology-oncology participants performed at least one activity with antineoplastic drugs. Nurses wore all of the recommended 
protection for technical activities (86.2\%), but rarely for non-technical activities (14.9\%). Pharmacy technicians and pharmacists wore all of the recommended protection for all activities (100.0\%).

Discussion: We implemented a pilot study of biological monitoring of workers with a good participation rate. The absence of positive samples showed the usefulness of maintaining good working practices. We found areas where the worker protection can be enhanced, especially for non-technical activities.

Conclusion: The absence of positive samples is a good indication that the measures that were put in place ensured workers' safety. Repeating the biological monitoring measures every few years could help confirm that the working practices are continuously followed by workers.

\section{Extended-Interval Dosing Recommendations for Gentamicin in Neonates \\ Specialties in Pharmacy Practice Award, sponsored by Pharmascience Inc.}

Potvin $M^{1}$, Walker $S A N^{1,2,3,4}$, Elligsen $M^{1}$, Iaboni $D^{5}$, Findlater $C^{5}$, Seto $W^{2,6}, N g E^{5,7}$

${ }^{1}$ Department of Pharmacy, Sunnybrook Health Sciences Centre, Toronto, ON ${ }^{2}$ Leslie L Dan Faculty of Pharmacy, University of Toronto, Toronto, ON ${ }^{3}$ Division of Infectious Diseases, Sunnybrook Health Sciences Centre, Toronto, ON

${ }^{4}$ Sunnybrook Health Sciences Centre Research Institute, Toronto, ON ${ }^{5}$ Women and Babies Program, Sunnybrook Health Sciences Centre, Toronto, $O N$

${ }^{6}$ Department of Pharmacy, The Hospital for Sick Children, Toronto, ON ${ }^{7}$ Department of Pediatrics, University of Toronto, Toronto, $O N$

Purpose/Objectives: To determine gentamicin pharmacokinetics in neonates, and develop initial $\mathrm{mg} / \mathrm{kg}$ dosing recommendations that optimize target peak and trough concentrations for extended-interval dosing (EID) regimens.

Description of the Methods: Steady-state gentamicin concentration data were retrospectively collected for 60 neonates admitted to a neonatal intensive care unit. Mean pharmacokinetics were calculated and multiple linear regression was performed to determine significant covariates of clearance and volume of distribution. A classification and regression tree (CART) analysis was used to identify breakpoints for significant covariates. Monte Carlo Simulation was subsequently used to determine optimal dosing recommendations for each CART-identified sub-group.

Project Results: Gentamicin clearance and volume of distribution were significantly associated with weight at gentamicin initiation. CARTidentified breakpoints for weight at gentamicin initiation were: $\leq 850 \mathrm{~g}$, $851-1200 \mathrm{~g}$, and $>1200 \mathrm{~g}$. No significant difference in pharmacokinetics was found between the $>1200 \mathrm{~g}$ and $851-1200 \mathrm{~g}$ sub-groups, due to inadequate sample size in the $>1200 \mathrm{~g}$ weight category. Monte Carlo Simulation identified that an $8-9 \mathrm{mg} / \mathrm{kg} / \mathrm{dose}$ of gentamicin administered every 72 hours in neonates weighing $\leq 850 \mathrm{~g}$, and every 48 hours in neonates weighing $851-1200 \mathrm{~g}$, provided the best probability of attaining EID targets.

Discussion: This study led to the development of practical, weight-based, initial gentamicin dosing nomograms with an excellent probability of target peak and trough attainment in a patient population for whom data is currently lacking.

Conclusion: This study provides initial recommendations for EID regimens in neonates weighing $\leq 1200 \mathrm{~g}$. Further studies are needed to prospectively evaluate these recommendations.

\section{Acute Kidney Injury with Tobramycin-Impregnated Bone Cement Spacers in Prosthetic Joint Infections Specialties in Pharmacy Practice Award, sponsored by Sandoz Canada Inc.}

Aeng ESY', Shalansky $K F^{1,2}$, Lau TTY $Y^{1,2,3}$, Zalunardo $N^{4}$, Li $G^{4}$, Bowie WR ${ }^{3}$, Duncan $C P^{5}$

${ }^{1}$ Faculty of Pharmaceutical Sciences, The University of British Columbia, Vancouver, $B C$

${ }^{2}$ Department of Pharmaceutical Sciences, Vancouver General Hospital, Vancouver, $B C$

${ }^{3}$ Division of Infectious Diseases, Faculty of Medicine, The University of British Columbia, Vancouver, $B C$

${ }^{4}$ Division of Nephrology, Vancouver General Hospital, Vancouver, BC

${ }^{5}$ Division of Orthopaedics, Faculty of Medicine, The University of British Columbia, Vancouver, BC

Introduction: Antibiotic-impregnated bone cement spacer (ACS) with tobramycin \pm vancomycin is commonly used in a two-stage replacement of infected prosthetic joints. This procedure has been associated with development of acute kidney injury (AKI). The objective of this study was to determine the incidence and risk factors for AKI after implantation of tobramycin-impregnated ACS.

Methods: This prospective, observational study evaluated 50 consecutive patients who received tobramycin ACS for first stage revision of an infected hip or knee arthroplasty from Aug 2011 to Feb 2013. AKI was defined as $50 \%$ or greater rise in serum creatinine (SCr) from baseline within the first 7 postoperative days.

Results: The incidence of AKI was 20\% with median onset occurring at POD 2 (interquartile range $[\mathrm{IQR}]=1-3$ ); patients with AKI had a longer median duration of hospital stay ( 16 days, $\mathrm{IQR}=12-17$, vs 10 days, $\mathrm{IQR}=8-10 ; \mathrm{p}=0.03)$. Serum tobramycin concentrations were significantly higher in the AKI group, peaking on POD 1 (median $1.9 \mathrm{mg} / \mathrm{L}$ vs $0.9 \mathrm{mg} / \mathrm{L}, \mathrm{p}=0.01$ ). Risk factors for nephrotoxicity identified by multivariate analysis were use of bone cement pre-manufactured with gentamicin $(\mathrm{OR}=8.2 ; 95 \% \mathrm{CI}=1.1-60, \mathrm{p}=0.04)$, administration of blood transfusions intraoperatively $(\mathrm{OR}=32.5 ; 95 \% \mathrm{CI}=2.3-454.3$; $\mathrm{p}=0.01)$ and non-steroidal anti-inflammatory drugs postoperatively (OR $=23.0 ; 95 \% \mathrm{CI}=1.3-397.7, \mathrm{p}=0.03$ ).

Discussion: We found a $20 \%$ incidence of AKI in patients with infected joint arthroplasties receiving tobramycin ACS which is comparable to other reports. AKI is a known risk factor for chronic kidney disease.

Conclusion: Tobramycin ACS for treatment of prosthetic joint infections is associated with a high risk of AKI. Measures to minimize AKI risk in the perioperative period include close monitoring of $\mathrm{SCr}$, and avoiding pre-manufactured bone cement containing gentamicin and potential nephrotoxins. 


\title{
2016 CSHP NATIONAL AWARDS PROGRAM / PROGRAMME NATIONAL DES PRIX 2016 DE LA SCPH
}

Learning from Medication Incidents One Group at a Time: The Development and Evaluation of Multi-Incident Analysis Workshops

Teaching, Learning and Education Award, sponsored by Eli Lilly Canada Inc.

Cheng R, Pan V, Carthy B, Yoo L, Ng K, Ho C, Yu J, Hyland S Institute for Safe Medication Practices Canada, Toronto, ON

Introduction: Learning from medication incidents is a fundamental step towards the advancement of safe medication practices. The multiincident analysis technique makes possible the analysis of medication incidents a group at a time and therefore facilitates the process of learning from medication incidents.

Purpose: The purpose of this project is to develop and evaluate the effectiveness of an education workshop teaching a step-wise approach for conducting a multi-incident analysis.

Description of the Project: A full day multi-incident analysis workshop was created included both didactic and hands-on components. A ten-question multiple-choice pre/post-test was developed to evaluate knowledge acquisition. A participant feedback survey was used to evaluate participant satisfaction. A post workshop follow-up survey was then administered to participants several months after the workshops to assess the application of the multi-incident analysis technique in practice.

Results: Eight workshops were delivered since the development of the multi-incident analysis workshop in January 2013 with 61 attendees from diverse professional backgrounds. 39 pairs of pre/post-test scores were obtained. The mean pretest score was 36.9\% and the mean post-test score was $75.6 \%(p<0.00001)$. In terms of participant feedback, mean scores ranged from 4.7 to 4.9 out of 5 in all aspects of the workshop. The post workshop follow-up survey demonstrated that out of eight respondents, five of them applied the techniques into their workplace and found the workshop to be beneficial in their organization.

Discussion: The evaluation results demonstrated a significant improvement in the participants' understanding and application of the multiincident analysis technique; as well as a high degree of participant satisfaction.

Conclusion: Multi-incident analysis is an innovative analysis technique that greatly enhances the efficiency and quality of medication incident analysis. The evaluation results suggest that our multi-incident analysis workshops were highly effective in teaching the key concepts and facilitating the application of this important analysis technique.

\section{Canadian Society of Hospital Pharmacists Société canadienne des pharmaciens d'hôpitaux}

\author{
Membership Year 2016-2017 \\ (July I, 2016 to June 30, 2017)
}

Membership renewal time is just around the corner.... We start taking renewals for the 2016-2017 year on April I.

Support your profession by remaining part of a vibrant and strongly-networked pharmacy organization. Together, we are the voice of hospital pharmacists, helping to shape the delivery of healthcare in Canada.

Renew today at my.cshp.ca!

Membership Enquiries: Please contact Membership Services

Tel.: (6|3) 736-9733 Ext. 222 | Fax: (6|3) 736-5660 | Email: membershipservices@cshp.ca

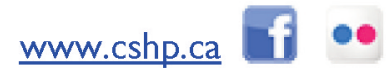

